\title{
Chronic venous disease among nurses in operating room and outside operating room
}

\author{
Prakaydao Kaima ${ }^{1,2}$, Termpong Reanpang ${ }^{3}$, Kanokwan Kulprachakarn ${ }^{1,4}$, Sasinat Pongtam¹, Kittipan \\ Rerkasem $^{1,3}$ \\ ${ }^{1}$ NCD Center of Excellence, Research Institute for Health Sciences, Chiang Mai University, Chiang Mai 50200, Thailand. \\ ${ }^{2}$ Institute of Research and Development Chiang Mai Rajabhat University, Mae Rim Campus, Chiang Mai 50180, Thailand. \\ ${ }^{3}$ Department of Surgery, Faculty of Medicine, Chiang Mai University, Chiang Mai 50200, Thailand. \\ ${ }^{4}$ School of Health Science Research, Research Institute for Health Sciences, Chiang Mai University, Chiang Mai 50200, Thailand.
}

Correspondence to: Prof. Kittipan Rerkasem, Division of Vascular and Endovascular Surgery, Department of Surgery, Faculty of Medicine, Chiang Mai University, Chiang Mai 50200, Thailand. E-mail: rerkase@gmail.com

How to cite this article: Kaima P, Reanpang T, Kulprachakarn K, Pongtam S, Rerkasem K. Chronic venous disease among nurses in operating room and outside operating room. Vessel Plus 2020;4:37. http://dx.doi.org/10.20517/2574-1209.2020.50

Received: 11 Sep 2020 First Decision: 9 Oct 2020 Revised: 26 Oct 2020 Accepted: 12 Nov 2020 Published: 26 Nov 2020

Academic Editor: Rene Gordon Holzheimer Copy Editor: Cai-Hong Wang Production Editor: Jing Yu

\begin{abstract}
Aim: Chronic venous disease (CVD) is very common in nurses. Noticeably, operating room (OR) nurses are predicted to have a major prevalence of varicose veins. We investigated whether the prevalence of CVD in OR nurses was more than non-OR nurses.
\end{abstract}

Methods: Study populations were OR nurses and non-OR nurses at the Faculty of Medicine, Chiang Mai University. Information was compiled by questionnaire. Physical examination was operated by examiners for CVD based on clinical finding using Comprehensive Classification System for Chronic Venous Disorders classification.

Results: 222 nurses were included. The prevalence of C0-C2 was notably different between the two groups ( $P$ $<0.001$ ). The prevalence of $C 1$ in OR nurses and non-OR nurses was $59.6 \%$ and $72.1 \%$ while the prevalence of C2 in OR nurses and non-OR nurses was $8.1 \%$ and $16.4 \%$, respectively. Nevertheless, the quality of life was not remarkably different between the two groups.

Conclusion: The results demonstrated that CVD in non-OR nurses appear to be higher than OR nurses.

Keywords: Chronic venous disease, operating room nurses, CEAP classification 


\section{INTRODUCTION}

Chronic venous disease (CVD) is a condition that effect the veins of the leg. It includes various clinical signs from mild to severe form such as telangiectasia, reticular vein, varicose vein, leg edema, hyperpigmented skin changes, dermal sclerosis, and venous ulcer. Telangiectasia and reticular veins are defined as the modest form of chronic venous disease. They are abnormalities of the vein inside or near the skin layer, which are swollen with blood accumulating to blue or purple lines, usually in the legs or feet. Varicose veins are larger than telangiectasia and are located below the skin layer and in subcutaneous fat. They are common, especially for women, elderly individuals, pregnant women, obese people, family history, and those who walk or stand for long periods of time, which gives fatigue, swelling, and other symptoms ${ }^{[1-5]}$. This disease usually does not cause serious complications such as death, but it can affect the quality of life.

This study focuses on nurses. Nurses typically have to walk or stand constantly, which results in an increased risk of developing CVD due to their working conditions ${ }^{[4-7]}$. There is often a debate in the nursing field that operating room nurses (OR nurses) walk and stand all day and are more likely to have varicose veins than other nurses (non-OR nurses). However, non-OR nurses have claimed that because they are constantly walking, they are more likely to have varicose veins. Thus far, there have been no studies to settle the debate. Therefore, our study will investigate the prevalence of chronic venous disease in OR nurses and non-OR nurses, as well as a comparative study between the two groups.

\section{METHODS}

\section{Patients and data collection}

A cross-sectional study was performed between May 2013 and June 2013. The study subjects were nurses including OR nurses and non-OR nurses at Faculty of Medicine, Chiang Mai University, Chiang Mai, Thailand. This study was approved by our local ethics committee, Faculty of Medicine, Chiang Mai University (SUR-13-1390-EX). All subjects provided written informed consent. This study was conducted per the guidelines of the Declaration of Helsinki. Data was collected by a questionnaire that was divided into two sections. The first section of the questionnaire pertained to individual characteristics, risk factors, and history of CVD. The second section pertained to quality of life by using Chronic Venous Insufficiency Quality of Life Questionnaire-14 (CIVIQ-14) that was validated as a useful measurement in assessing $\mathrm{CVD}^{[8]}$. The physical examination was operated by the examiners for the varicose vein based on the clinical finding using CEAP classification. The CEAP classification includes clinical, etiologic, anatomic, and pathologic parts ${ }^{[9]}$. The clinical classification consists Co to C6. Co is no visible sign of CVD. C1 is telangiectasia or reticular veins. $\mathrm{C} 2$ is varicose veins. $\mathrm{C} 3$ is leg edema. $\mathrm{C} 4$ is hyperpigmented skin or dermal sclerosis. $\mathrm{C} 5$ is healed venous ulcer and $\mathrm{C} 6$ is active venous ulcer.

\section{Statistical analysis}

All statistical analyses were performed with STATA 14.0 (StataCorp LP, USA) for Windows. Descriptive statistics of continuous variables were represented using mean \pm standard deviation (SD). Categorized variables were represented with percentages. Differences between the two groups were analyzed with T-test/ Mann Whitney $U$ test or Chi-square. Statistical significance was set at $P<0.05$. Sample size calculation for this study were based on the possible proportion of varicose veins from $30 \%$ to $35 \%{ }^{[4]}$. Significance levels are $5 \%$ and power levels are $80 \%$.

\section{RESULTS}

A total of 222 nurses were included in this study. Two hundred and nine (94.1\%) nurses were female, and 99 (44.6\%) were OR nurses. The baseline characteristics are shown in Table 1. We found that the height of the OR nurses was greater than that of the non-OR nurses $(155.61 \pm 5.67 \mathrm{~cm} v s .157 .77 \pm 6.05 \mathrm{~cm} ; P=0.013)$. 
Table 1. Baseline characteristics and comparison between non-OR nurses and OR nurses

\begin{tabular}{|c|c|c|c|c|}
\hline & Total $n=222$ & Non-OR nurses $n=123$ & OR nurses $n=99$ & $P$-value \\
\hline Gender, n (\%) & & & & 0.066 \\
\hline Male & $13(5.9)$ & $4(3.3)$ & $9(9.1)$ & \\
\hline Female & $209(94.1)$ & 119 (96.7) & $90(90.9)$ & \\
\hline \multicolumn{5}{|l|}{ Age (years), $n(\%)$} \\
\hline$<35$ & $36(16.5)$ & $13(10.8)$ & $23(23.5)$ & 0.094 \\
\hline $35-40$ & $37(17.0)$ & $22(18.3)$ & $15(15.3)$ & \\
\hline $41-50$ & $82(37.6)$ & $47(39.2)$ & $35(35.7)$ & \\
\hline$>50$ & $63(28.9)$ & $38(31.7)$ & $25(25.5)$ & \\
\hline Mean \pm SD & $44.36 \pm 9.57$ & $45.80 \pm 8.49$ & $42.60 \pm 10.51$ & 0.067 \\
\hline Weight (kg) & & & & \\
\hline Mean \pm SD & $56.36 \pm 9.19$ & $56.09 \pm 8.01$ & $56.69 \pm 10.49$ & 0.829 \\
\hline Height (cm) & & & & \\
\hline Mean \pm SD & $156.58 \pm 5.92$ & $155.61 \pm 5.67$ & $157.77 \pm 6.05$ & $0.013^{\star}$ \\
\hline $\begin{array}{l}\mathrm{BMI}\left(\mathrm{kg} / \mathrm{m}^{2}\right) \\
\text { Mean }+\mathrm{SD}\end{array}$ & $22.99+3.51$ & $23.18+3.27$ & $22.76 \pm 3.78$ & 0.224 \\
\hline Standing (h/day) & & & & \\
\hline Mean \pm SD & $6.03 \pm 2.58$ & $6.14 \pm 2.64$ & $5.60 \pm 2.52$ & 0.459 \\
\hline Sitting (h/day) & & & & \\
\hline Mean \pm SD & $4.45 \pm 2.61$ & $4.72 \pm 2.72$ & $4.11 \pm 2.45$ & 0.099 \\
\hline Exercise & & & & 0.212 \\
\hline Yes & $111(50.9)$ & $56(47.1)$ & $55(55.6)$ & \\
\hline No & $107(49.1)$ & $63(52.9)$ & $44(44.4)$ & \\
\hline Family history of varicose veins & & & & 0.167 \\
\hline Yes & $49(22.1)$ & $32(26.0)$ & $17(17.2)$ & \\
\hline No & $173(77.9)$ & $91(74.0)$ & $82(82.8)$ & \\
\hline
\end{tabular}

N: number; BMI: body mass index; SD: standard deviation. ${ }^{\star} P$ value $<0.05$ was considered statistically significant

However, we found that the body mass index (BMI), weight, standing hours, sitting hours, exercise, and family history of varicose veins to be similar in both nurses.

Concerning the severity of clinical CEAP classification in nurses, the most frequent stages were $\mathrm{C} 1$ (66.5\%), Co $(20.8 \%)$, and C2 (12.7\%). The prevalence of Co-C2 was notably different between the two groups $(P<$ $0.001)$ as shown in Figure 1. The prevalence of $\mathrm{C} 1$ in OR nurses and non-OR nurses was $59.6 \%$ and $72.1 \%$, respectively, while the prevalence of $\mathrm{C} 2$ in OR nurses and non-OR nurses was $8.1 \%$ and $16.4 \%$, respectively. Nevertheless, the quality of life was not remarkably different $(85.75 \pm 11.91$ vs. $85.60 \pm 12.24 ; P=0.962)$ between the two groups, as show in Table 2.

\section{DISCUSSION}

The important finding in this study is that the prevalence of $\mathrm{C} 2$ and $\mathrm{C} 1$ in non-OR nurses was significantly higher than OR nurses. Particularly, $\mathrm{C} 2$ in non-OR nurses was two times more frequent than in OR nurses. Telangiectasia and reticular veins are the most common findings of chronic venous disorder in the general population. A study of 5,187 people in Italy found that $64.8 \%$ had telangiectasia ${ }^{[10]}$. Our study had similar results. C1 was the most common finding of clinical sign; the average prevalence was $66.5 \%$. As we know, nursing is a career that involves standing for a long time at work. Prolonged standing is one of the risk factors of $\mathrm{CVD}^{[1,2]}$. Some studies showed that prolonged standing was found to be a significant factor for varicose veins among nurses at Dhulikhel Hospital and a university hospital in Busan, South Korea ${ }^{[6,7]}$. The standing position results in high venous pressure or venous hypertension in the veins of the leg. Particularly, persons who stand still in the same position for long periods of time are exposed to venous hypertension, which when combined to valvular incompetence or muscle pump dysfunction can aggravate this disease ${ }^{[11]}$. Bahk et al. ${ }^{[1]}$ demonstrated that prolonged standing time was associated with varicose veins and nocturnal leg cramps. However, the standing time per day in our study is not different between the two groups of nurses. The mean standing time per day of OR nurses is $5.6 \mathrm{~h}$ and non-OR nurses is $6.14 \mathrm{~h}$. 


\section{Prevalence of CEAP classification}

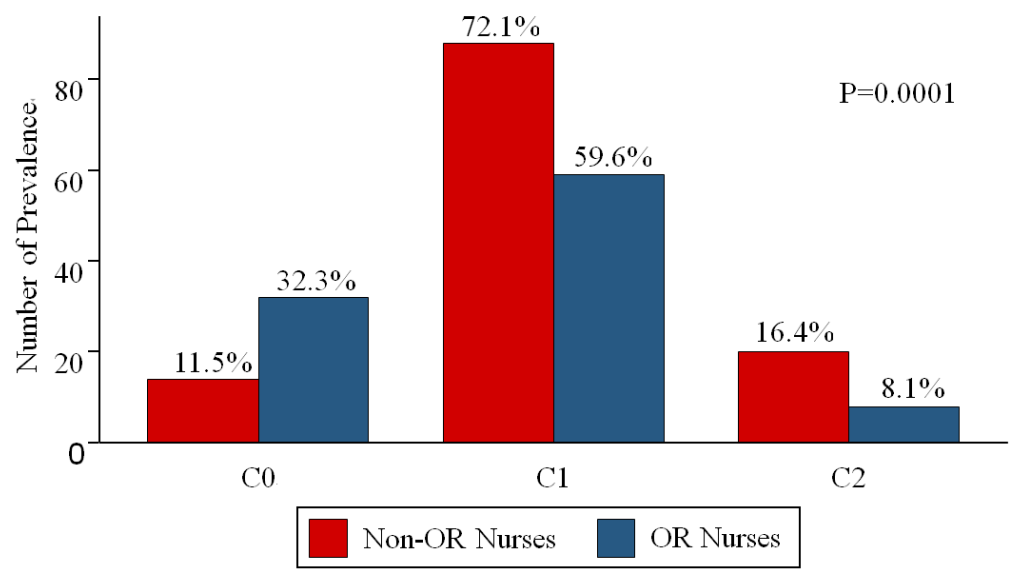

Figure 1. Frequency and percentage of clinical CEAP intensity in nurses. CEAP: Comprehensive Classification System for Chronic Venous Disorders

Table 2. Clinical CEAP classification and CIVIQ comparison between non-OR nurses and OR nurses

\begin{tabular}{llcll}
\hline & Total $\boldsymbol{n}=\mathbf{2 2 2}$ & Non-OR nurses $\boldsymbol{n}=\mathbf{1 2 3}$ & OR nurses $\boldsymbol{n} \mathbf{9 9}$ & $\boldsymbol{P}$-value \\
\hline CEAP, $n(\%)$ & & & & $<0.001^{\star}$ \\
CO & $46(20.8)$ & $14(11.5)$ & $32(32.3)$ & \\
C1 & $147(66.5)$ & $88(72.1)$ & $59(59.6)$ & \\
C2 & $28(12.7)$ & $20(16.4)$ & $8(8.1)$ & 0.962 \\
CIVIQ & & & & \\
Mean \pm SD & $85.69 \pm 12.04$ & $85.75 \pm 11.91$ & $85.60 \pm 12.24$ & \\
Median (min-max) & $89(30-100)$ & $89(43-100)$ & $88(30-100)$ & \\
\hline
\end{tabular}

N: number; CEAP: Comprehensive Classification System for Chronic Venous Disorders; CIVIQ: Chronic Venous Insufficiency Quality of Life Questionnaire-14. ${ }^{\star} P$ value $<0.05$ was considered statistically significant

Other risk factors of chronic venous disease such as older age, female gender, obesity, and family history of varicose veins are not different between the groups. The height of the OR nurses is greater than non-OR nurses and have statistical significance, but perhaps the difference around two centimeters might not have any clinical relevance. In short, we found that non-OR nurses had more prevalence of CVD, although our study did not show risk factor differences. The reason behind this difference is not clear. Further cohort study is needed to continue for more specific and further study risk factors. This can help nurses improve both their physical and mental health.

There were limitations in this study. The OR nurse group has a greater frequency of younger nurses (double frequency in the below 35 group) than those in non-OR nurse group. Also, there were more males in OR nurse group than those in non-OR group. As is well known, the prevalence of CVD is age and gender dependent. These might be a bias of this study. There are a large number of risk factors that can determine the progression in CVD including weight, body mass index, number of pregnancy, career, and family history of chronic venous disease. Therefore, our findings need to be confirmed in a large population with adjusting confounders (multivariate analysis). Additionally, this study was a cross sectional study, so it cannot explain cause and effect.

CVD has a substantial effect on the quality of life, especially physical health aspects ${ }^{[12,13]}$. The mental health aspect is affected only in a severe stage $(\mathrm{C} 3 \text { or more })^{[14]}$. Our study shows non-OR nurses had more CVD (C1 and $\mathrm{C} 2)$, but the quality of life was not remarkably different $(P=0.962)$. 
In conclusion, $\mathrm{CVD}$ in non-OR nurses seems to be more common than in OR nurses; however, the quality of life was not different between the two groups.

\section{DECLARATIONS}

Acknowledgments

We would like to thank all staff in OPD101 at Maharaj Nakorn Chiang Mai Hospital, Chiang Mai, Thailand. This study was supported by Chiang Mai University.

\section{Authors' contributions}

Made substantial contributions to conception and design of the study and performed data analysis and interpretation: Kaima P, Reanpang T, Rerkasem K

Performed data acquisition, as well as provided administrative, technical, and material support: Kaima P, Kulprachakarn K, Pongtam S

\section{Availability of data and materials}

Not applicable.

\section{Financial support and sponsorship}

This work was supported by Chiang Mai University.

\section{Conflicts of interest}

All authors declared that there are no conflicts of interest.

\section{Ethical approval and consent to participate}

The ethics committee of Faculty of Medicine, Chiang Mai University approved this study (SUR-13-1390EX). All subjects provided written informed consent. This study was conducted as per guidelines of the Declaration of Helsinki.

\section{Consent for publication}

Not applicable.

\section{Copyright}

(c) The Author(s) 2020.

\section{REFERENCES}

1. Bahk JW, Kim H, Jung-Choi K, Jung MC, Lee I. Relationship between prolonged standing and symptoms of varicose veins and nocturnal leg cramps among women and men. Ergonomics 2012;55:133-9.

2. Beebe-Dimmer JL, Pfeifer JR, Engle JS, Schottenfeld D. The epidemiology of chronic venous insufficiency and varicose veins. Ann Epidemiol 2005;15:175-84.

3. Joseph N, B A, Faizan Thouseef M, Devi M U, Abna A, Juneja I. A multicenter review of epidemiology and management of varicose veins for national guidance. Ann Med Surg (Lond) 2016;8:21-7.

4. Mishra N, Solanki SL, Mishra S. Lower limb varicose veins among nurses: a cross sectional study in Udaipur. Int $J$ Cur Res Rev 2015;7:51-5.

5. Sharif Nia H, Chan YH, Haghdoost AA, Soleimani MA, Beheshti Z, Bahrami N. Varicose veins of the legs among nurses: occupational and demographic characteristics. Int J Nurs Pract 2015;21:313-20.

6. Shakya R, Karmacharya RM, Shrestha R, Shrestha A. Varicose veins and its risk factors among nurses at Dhulikhel hospital: a cross sectional study. BMC Nurs 2020;19:8.

7. Yun MJ, Kim YK, Kang DM, et al. A study on prevalence and risk factors for varicose veins in nurses at a university hospital. Saf Health Work 2018;9:79-83.

8. Launois R, Reboul-Marty J, Henry B. Construction and validation of a quality of life questionnaire in chronic lower limb venous insufficiency (CIVIQ). Qual Life Res 1996;5:539-54.

9. Porter JM, Moneta GL; An International Consensus Committee on Chronic Venous Disease. Reporting standards in venous disease: an 
update. J Vasc Surg 1995;21:635-45.

10. Chiesa R, Marone EM, Limoni C, Volonté M, Schaefer E, Petrini O. Chronic venous insufficiency in Italy: the 24-cities cohort study. Eur J Vasc Endovasc Surg 2005;30:422-9.

11. Raffetto JD, Mannello F. Pathophysiology of chronic venous disease. Int Angiol 2014;33:212-21.

12. Kaplan RM, Criqui MH, Denenberg JO, Bergan J, Fronek A. Quality of life in patients with chronic venous disease: San Diego population study. J Vasc Surg 2003;37:1047-53.

13. Kurz X, Lamping DL, Kahn SR, et al; VEINES Study Group. Do varicose veins affect quality of life? Results of an international population-based study. J Vasc Surg 2001;34:641-8.

14. Andreozzi GM, Cordova RM, Scomparin A, Martini R, D’Eri A, Andreozzi F. Quality of life in chronic venous insufficiency: an Italian pilot study of the Triveneto Region. Int Angiol 2005;24:272-7. 